\title{
Bounds on relative entropy of entanglement for multi-party systems
}

\author{
M. B. Plenio ${ }^{1}$ and V. Vedral ${ }^{1,2}$ \\ 1 Optics Section, The Blackett Laboratory, Imperial College, London SW7 2BW, UK \\ ${ }^{2}$ Centre for Quantum Computation, Clarendon Laboratory, Univ. of Oxford, Oxford OX1 3PU,UK
}

We present upper and lower bounds to the relative entropy of entanglement of multi-party systems in terms of the bi-partite entanglements of formation and distillation and entropies of various subsystems. We point out implications of our results to the local reversible convertibility of multi-party pure states and discuss their physical basis in terms of deleting of information.

\section{INTRODUCTION}

The quantification of entanglement is a long standing problem in quantum information theory [1]. Early work in this field focused on bi-partite entanglement, but in this paper we address the problem of quantifying entanglement for multi-party systems. This is an interesting and complex issue since it is not always evident how the existing measures for bi-partite systems can be generalized to the multi-partite case. The three most promising ideas for quantifying entanglement of multi-party systems are the entanglement of distillation [2], the entanglement of formation [2] and the relative entropy of entanglement [5 9]. In the bi-partite setting, the meaning of both entanglement of formation and the entanglement of distillation is quite clear. However, these two measures do not have an entirely straightforward meaning when one considers mulit-partite entanglement. Let us consider the entanglement of distillation first. This would be the number of "maximally" entangled states that the parties could distill asymptotically by local operations and classical communication (LOCC) from a given multi-party state. The central issue here is the definition of maximally entangled states, i.e. to what maximally entangled states should one distill to. In the multi-partite setting this question has not found an answer yet, and it is indeed not clear what this answer would be. In the bi-partite case a maximally entangled state is the one that allows to obtain any other pure state with certainty by LOCC and, in fact, it can be shown that the conversion is asymptotically reversible. For more than two qubits no such single pure state exists and it is unknown whether there is a finite set of states with this property [10-12]. For three qubit pure states one may believe that the minimal set from which every other pure state can be generated reversibly consists of GHZ and EPR states and it would then be reasonable to denote this set as the 'maximally entangled set' to which to which everything else should be distilled. However, it is not known whether the set of GHZ and EPR states is indeed sufficient to generate any other pure state asymptotically reversibly and there are indications that further states have to be added [13]. The same issues appear when we talk about the entanglement of formation. This measure tells us how many maximally entangled state the parties have to share a priori to be able to asymptotically create a given mixed state by LOCC.

In general, we would hope that at least for pure states there is a minimal reversible entanglement generating set (MREGS), to which all multi-parti states can be distilled and from which all of them can be reversibly created. As these sets are currently unknown and may be very complex, we would like to avoid them and instead use the relative entropy of entanglement. This measure can easily be generalised to multi-party states. Since for bi-partite systems the relative entropy of entanglement is an upper bound to distillable entanglement we would expect this to be the case in general.

We now proceed to show that this intuition is indeed correct and derive various upper and lower bounds of the relative entropy of entanglement for general multi-party states in terms of the bi-partite entanglements of formation and distillation and entropies of various subsystems. We discuss the physical meaning of our bounds in terms of deleting information and present some relevant applications.

\section{THE RELATIVE ENTROPY AND ENTANGLEMENT: DEFINITIONS AND USEFUL FORMULAE}

In this paper we will employ a particular measure of entanglement which is commonly called the relative entropy of entanglement [5] 9. This measure can be defined for an arbitrary number of parties by the following formula

$$
E(\sigma)=\min _{\rho \in \mathcal{D}} S(\sigma \| \rho)
$$

where $\mathcal{D}$ is a set of disentangled (separable) states and where $S(\sigma \| \rho)=\operatorname{tr}\{\sigma \log \sigma-\sigma \log \rho\}$ is the quantum relative entropy [15]. For the purpose of this paper, we assume that $\mathcal{D}$ is the set of the states that can be created locally, i.e. it is fully separable. Also, by $E_{n}(\sigma)$ we will always denote the relative entropy of entanglement for n-party systems with respect to the set of fully separable states.

Many of the results in this paper are based on the following inequality [9] that holds true for any state $\sigma_{A B}$ 
and any separable state $\rho_{A B}$ of two parties.

$$
S\left(\sigma_{A B} \| \rho_{A B}\right)-S\left(\sigma_{A} \| \rho_{A}\right) \geq S\left(\sigma_{A}\right)-S\left(\sigma_{A B}\right)
$$

This inequality can be generalized directly to multipartite entangled systems. For tri-partite systems, for example, we find that for any state $\sigma_{A B C}$ and any triseparable state $\rho_{A B C}$, the following inequality

$$
S\left(\sigma_{A B C} \| \rho_{A B C}\right)-S\left(\sigma_{A B} \| \rho_{A B}\right) \geq S\left(\sigma_{A B}\right)-S\left(\sigma_{A B C}\right)
$$

is satisfied. We now show how to use this result to derive upper and lower bounds on the relative entropy of entanglement of many-party states.

\section{BOUNDS ON THE RELATIVE ENTROPY OF ENTANGLEMENT}

The aim of this section is the derivation of upper and lower bounds of the relative entropy of entanglement for $n$ parties in terms of the relative entropies for smaller numbers of subsystems. We begin by considering pure tri-partite states and later generalize the results to the case of arbitrarily many subsystems.

Theorem 1: For any pure tri-partite states $\sigma_{A B C}$ we find

$$
\begin{aligned}
& \max \left\{E_{2}\left(\sigma_{A B}\right)+S\left(\sigma_{A B}\right), E_{2}\left(\sigma_{A C}\right)+S\left(\sigma_{A C}\right), E_{2}\left(\sigma_{B C}\right)+S\left(\sigma_{B C}\right)\right\} \leq E_{3}\left(\sigma_{A B C}\right) \\
& E_{3}\left(\sigma_{A B C}\right) \leq \min \left\{S\left(\sigma_{A}\right)+S\left(\sigma_{B}\right), S\left(\sigma_{A}\right)+S\left(\sigma_{C}\right), S\left(\sigma_{B}\right)+S\left(\sigma_{C}\right)\right\}
\end{aligned}
$$

Proof: We begin with the proof of the first inequality. We will employ the fact that $E_{3}$ is an entanglement monotone, i.e. it does not increase under local operations. Consider now three parties A, B and C that wish to create an arbitrary pure quantum state $\sigma_{A B C}$. One possible procedure is that Alice creates the state locally and then compresses the particles that should go to Bob (with the efficiency $S\left(\sigma_{B}\right)$ ) and those that should go to Charles (with the efficiency $S\left(\sigma_{C}\right)$ ) and then uses $S\left(\sigma_{B}\right)$ shared singlets between herself and Bob and $S\left(\sigma_{C}\right)$ shared singlets between herself and Charles to teleport these particles to them. Therefore they have consumed $S\left(\sigma_{C}\right)+S\left(\sigma_{B}\right)$ ebits in total to create this state. As we know that $E_{3}$ is an entanglement monotone, we find

$$
E_{3}\left(\sigma_{A B C}\right) \leq S\left(\sigma_{B}\right)+S\left(\sigma_{C}\right) .
$$

Permuting the indices cyclically we find the sharpest bound

$$
\begin{aligned}
E_{3}\left(\sigma_{A B C}\right) \leq & \min \left\{S\left(\sigma_{A}\right)+S\left(\sigma_{B}\right),\right. \\
& \left.S\left(\sigma_{A}\right)+S\left(\sigma_{C}\right), S\left(\sigma_{B}\right)+S\left(\sigma_{C}\right)\right\} .
\end{aligned}
$$

Let us now proceed to prove the second inequality. This can easily be performed using Eq. (2). Given a pure state $\sigma_{A B C}$, we obtain from this inequality that

$$
S\left(\sigma_{A B C} \| \rho_{A B C}\right) \geq S\left(\sigma_{A B} \| \rho_{A B}\right)+S\left(\sigma_{A B}\right) .
$$

If we replace $\rho_{A B C}$ by the closest tri-separable state to $\sigma_{A B C}$ which we denote by $\rho_{A B C}^{*}$ then we find

$$
E_{3}\left(\sigma_{A B C}\right)=S\left(\sigma_{A B C} \| \rho_{A B C}^{*}\right) \geq S\left(\sigma_{A B} \| \rho_{A B}^{*}\right)+S\left(\sigma_{A B}\right)
$$

where $\rho_{A B}^{*} \equiv \operatorname{tr}_{C}\left\{\rho_{A B C}^{*}\right\}$. As $\rho_{A B}^{*}$ is evidently separable we immediately have that $S\left(\sigma_{A B} \| \rho_{A B}^{*}\right) \geq E_{2}\left(\sigma_{A B}\right)$ so that

$$
E_{3}\left(\sigma_{A B C}\right)=S\left(\sigma_{A B C} \| \rho_{A B C}^{*}\right) \geq E_{2}\left(\sigma_{A B}\right)+S\left(\sigma_{A B}\right)
$$

If we permute the indices cyclically we get three inequalities and obtain the sharpest bound

$$
\begin{aligned}
E_{3}\left(\sigma_{A B C}\right) & \geq \max \left\{E_{2}\left(\sigma_{A B}\right)+S\left(\sigma_{A B}\right), E_{2}\left(\sigma_{A C}\right)\right. \\
& \left.+S\left(\sigma_{A C}\right), E_{2}\left(\sigma_{B C}\right)+S\left(\sigma_{B C}\right)\right\} \square .
\end{aligned}
$$

Corollary: For any pure tri-partite states $\sigma_{A B C}$ we find

$$
\frac{2}{3}\left(S\left(\sigma_{A}\right)+S\left(\sigma_{B}\right)+S\left(\sigma_{C}\right)\right) \geq E_{3}\left(\sigma_{A B C}\right) \geq \frac{1}{3}\left(E_{2}\left(\sigma_{A B}\right)+E_{2}\left(\sigma_{A B}\right)+E_{2}\left(\sigma_{A B}\right)+\frac{1}{3}\left(S\left(\sigma_{A}\right)+S\left(\sigma_{B}\right)+S\left(\sigma_{C}\right)\right)\right.
$$

Proof: The Corollary follows directly from the inequalities stated in the theorem by taking the average over all combinations of two parties $\square$.

One may wonder for which states the bounds presented in the theorem and the corollary are saturated. While we do not have a general answer to this question, it is straightforward to show that both inequalities 1 and 5 are saturated for GHZ-like states $(\alpha|000\rangle+\beta|111\rangle$ or local unitary transformations) and any bi-partite pure state (where the third subsystem is disentangled from the first two). For the W-state given by $|W\rangle=(|100\rangle+|010\rangle+$ $|001\rangle) / \sqrt{3}$ we find that the lower bound 5 is saturated 
only as we find $E_{3}(W)=2 \log 3-2$ [14. In fact for all states of the form $|\psi\rangle=e|100\rangle+f|010\rangle+f|001\rangle[13] \mathrm{e}$ find that the upper bound 5 is not saturated .

It would be interesting to know whether one can sharpen the upper bound presented in the corollary can be sharpened further to

$$
E_{3}\left(\sigma_{A B C}\right) \leq \frac{1}{2}\left(S\left(\sigma_{A}\right)+S\left(\sigma_{B}\right)+S\left(\sigma_{C}\right)\right)
$$

We have no counter-example but no proof of this conjecture either. It is, however, clear that the coefficient in front of the entropies cannot be sharpened further because this upper bound is saturated by, for example, EPR states and GHZ states.

The inequalities we have presented here for tri-partite systems generalize straightforwardly to more than three parties. This is obtained by first generalizing the inequality 3 to $n$ systems and then following the steps leading up to theorems 1 and 5 .

\section{REVERSIBLE ENTANGLEMENT MANIPULATION AND THE RELATIVE ENTROPY OF ENTANGLEMENT}

It is interesting to note that a small modification of the lower bound presented in Theorem 1 actually becomes an equality for pure multi-partite states under some further assumptions which we review now. First of all we will have to replace the relative entropy of entanglement by its regularized version, ie.

$$
E_{3}^{\infty}(\sigma)=\lim _{n \rightarrow \infty} \frac{E_{3}\left(\sigma^{\otimes n}\right)}{n} .
$$

Furthermore let us make the (unproven) assumption that that the set of GHZ and the three possible EPR's forms what we called a MREGS. If this is so, then the following equations must be satisfied 11

$$
\begin{aligned}
S\left(\sigma_{A}\right) & =g+s_{A B}+s_{A C} \\
S\left(\sigma_{B}\right) & =g+s_{A B}+s_{B C} \\
S\left(\sigma_{C}\right) & =g+s_{A C}+s_{B C} \\
E_{2}^{\infty}\left(\sigma_{A B}\right) & =s_{A B} \\
E_{3}\left(\sigma_{A B C}\right) & =g+s_{A B}+s_{A C}+s_{B C}
\end{aligned}
$$

where $g$ is the number of GHZs and $s_{A B}$ is the number of singlets between A and B and so on. For the derivation of the first four equations see [11] and |13 for a slight modification. The last equality follows from the fact that $E_{3}^{\infty}$ is an entanglement monotone. Given these equations we immediately find that

$$
\begin{aligned}
E_{3}^{\infty}\left(\sigma_{A B C}\right)= & \frac{1}{3}\left(E_{2}^{\infty}\left(\sigma_{A B}\right)+E_{2}^{\infty}\left(\sigma_{A B}\right)+E_{2}^{\infty}\left(\sigma_{A B}\right)\right) \\
& +\frac{1}{3}\left(S\left(\sigma_{A}\right)+S\left(\sigma_{B}\right)+S\left(\sigma_{C}\right)\right)
\end{aligned}
$$

This equality has an interesting physical interpretation. Namely, it states that the entanglement of three subsystems is equal to the entropy due to deleting one of the subsystems plus the remaining entanglement between the other two subsystems, finally averaged over all three subsystems. This interpretation of multi-party entanglement is interesting because it combines the idea of the persistent entanglement due to loss of classical information [16,17 with Landauer's notion of deleting information and increasing entropy [18]. The persistent entanglement tells us how much entanglement is left when we erase information in a particle and Landauer's erasure tells us the entropy increase due to this erasure. Their sum gives the total entanglement.

\section{DISCUSSION AND CONCLUSIONS}

We have discussed in this paper how to generalize the relative entropy of entanglement to states involving more than two parties. Furthermore we have argued that this measure has a very natural generalization to multi-party states, while the entanglement of formation and the entanglement of distillation cannot be so easily generalized. In spite of this advantage of the relative entropy of entanglement, however, it is still very difficult to compute this quantity and no 'closed formula' exists even for bipartite states. It is therefore very important to provide upper and lower bounds for the relative entropy of entanglement which is the main result of this paper. We have also discussed the states which saturate either the upper or the lower bound. Finally, we have discussed the circumstances under which the lower bound becomes an equality and related this to Landauer's notion of information deletion and entropy increase.

\section{ACKNOWLEDGMENTS}

We would like to thank E.F. Galvao and L. Henderson for useful discussions. We are grateful to the Erwin Schrödinger Institute and Gasthof Hopferl in Vienna for their hospitality during our visit when this work was performed. We acknowledge financial support from the Erwin Schrödinger Institute, Elsag-Bailey, EPSRC, Hewlett-Packard, The Leverhulme Trust, and the EQUIP programme of the European Union.

[1] M.B. Plenio and V. Vedral, Contemp. Phys. 39, 431 (1998). 
[2] C. H. Bennett, H. J. Bernstein, S. Popescu, B. Schumacher, Phys. Rev. A 53, 2046 (1996).

[3] C.H. Bennett, D. DiVincenzo, J. Smolin and W.K. Wootters, Phys. Rev. A 53, 3824 (1996).

[4] W.K. Wootters, Phys. Rev. Lett. 80, 2245 (1998).

[5] V. Vedral, M.B. Plenio, M.A. Rippin, and P.L. Knight, Phys. Rev. Lett. 78, 2275 (1997).

[6] V. Vedral, M.B. Plenio, K. Jacobs and P.L. Knight, Phys. Rev. A 58, 4452 (1997).

[7] V. Vedral and M. B. Plenio, Phys. Rev. A 57, 1619 (1998).

[8] V. Vedral, Phys. Lett. A 262, 121 (1999).

[9] M.B. Plenio, S. Virmani and P. Papadopoulos, J. Phys. A 33, L193 (2000).

[10] C. H. Bennett, S. Popescu, D. Rohrlich, J. A. Smolin, A. V. Thapliyal, Los Alamos e-print quant-ph/9908073.
[11] N. Linden, S. Popescu, B. Schumacher, M. Westmoreland, Los Alamos e-print quant-ph/9912039.

[12] S. Wu and Y. Zhang, Los Alamos e-print quant$\mathrm{ph} / 0004020$.

13] E.F. Galvao, M.B. Plenio and S. Virmani, quantph/0008089, accepted for publication in J. Phys. A.

[14] E.F. Galvao and L. Henderson, private communication of a numerical result.

[15] A. Wehrl, Rev. Mod. Phys. 50, 221 (1978).

[16] J. Eisert, T. Felbinger, P. Papadopoulos, M.B. Plenio, and M. Wilkens. Phys. Rev. Lett. 84, 1611 (2000).

[17] L. Henderson and V. Vedral, Phys. Rev. Lett. 84, 2263 (2000).

[18] V. Vedral, Proc. Roy. Soc. London A 456, 969 (2000); M. B. Plenio, Phys. Lett. A 263, 281 (1999). 